\title{
ITERATED ROLES: MATHEMATICS AND APPLICATION
}

\author{
Stephen P. BORGATTI * \\ University of California, Irvine \\ John BOYD * \\ University of California, Irvine
}

\section{Martin EVERETT **}

Thames Polytechnic

\begin{abstract}
Recent work by Borgatti and Everett (1989) has shown that the collection of regular equivalences described by White and Reitz (1983) forms a lattice. In this paper, we present a procedure called iterated roles for tracing systematic paths through the lattice. At the heart of iterated roles is the proof that the regular equivalence of a regular equivalence is itself regular. The procedure enables us to find several otherwise unknown regular equivalences, including an extension of automorphic equivalence (Everett 1985) that is not sensitive to degree. A key benefit of iterated roles is the generation of sequences of hierarchically nested equivalences. This capability suggests an approach to role structure analysis in which one examines not just one blocking of actors but a series of increasingly broad simplifications of the data. Consequently, we are able to (a) choose the level of simplification that proves most illuminating, and (b) view both to broad structural outlines of the data and the finer details simultaneously.
\end{abstract}

\section{Introduction}

Since 1971, when Lorrain and White introduced structural equivalence, a variety of formal mathematical models of social role have been advanced. For the most part, these various models have been thought of as alternatives or successors to each other: when introducing a new model, authors have typically surveyed the existing models, pointed out their weaknesses, and presented their own model as a solution. For example, in 1978, Sailer criticized structural equivalence for requiring

* School of Social Science, University of California, Irvine CA 92717, U.S.A.

** School of Mathematics, Statistics and Computing, Thames Polytechnic, Wellington Street, London SE18 6PF, U.K. 
that equivalent actors be related to the same others, and introduced an alternative concept called structural relatedness. Later, White and Reitz (1983) refined Sailer's model and gave us regular equivalence. Similarly, Winship and Mandel (1983) introduced role similarity, Winship (1974) and Everett (1985) introduced automorphic equivalence, Breiger and Pattison (1986) developed local role algebras, and so on.

Recently, however, some progress has been made in the study of the relationships between many of these measures (Borgatti and Everett 1989). We know, for example, that the definition of regular equivalence describes a collection (in fact, a lattice) of role equivalences, of which structural equivalence, automorphic equivalence, REGE equivalence (White and Reitz 1984), and, with minor modifications, Winship and Mandel equivalence, are all members. The list is not exhaustive: even very small graphs may contain a very large number of distinct regular equivalences, each one a potential model of the role structure of the graph. Borgatti and Everett provide an algorithm (known as REGE/A) for generating many of these equivalences, but it is not well-suited to systematic, open-ended exploration of the lattice.

In this paper, we present a procedure for tracing systematic paths through the lattice. The procedure, called iterated roles, generates new regular equivalences, including an extension of automorphic equivalence that is not sensitive to degree. A key feature of iterated roles is the generation of sequences of hierarchically nested equivalences. This capability suggests a new approach to role analysis in which one examines not just one blocking of actors, but a series of increasingly broad simplifications of the data, thus allowing the analyst to (a) choose the level of simplification desired, and (b) simultaneously view both the broad structural outlines of the data and the finer details.

\section{Notation}

Let $G$ be a labelled graph with vertex set $V$ and edge set $E$. An automorphism of $G$ is a permutation $\pi$ of $V$ such that $(a, b) \in E$ iff $(\pi(a), \pi(b)) \in E$.

Definition. Two vertices $a, b \in V$ are structurally equivalent iff the permutation $(a, b)$ is an automorphism of $G$.

For more information on structural equivalence, the reader is referred to Lorrain and White (1971). 
Definition. Vertices $a, b \in V$ are automorphically equivalent iff there exists an automorphism $\pi$ such that $\pi(a)=b$.

For more information on automorphic equivalence, the reader is referred to Everett (1985), Everett and Borgatti (1988), and Boyd and Everett (1988).

Definition. If $\equiv$ is an equivalence relation on $V$ then $\equiv$ is a regular equivalence iff for all $a, b, c \in V, a \equiv b$ implies that if (i) $a R c$ then there exists $d \in V$ such that $b R d$ and $d \equiv c$, (ii) $c R a$ then there exists $d \in V$ such that $d R b$ and $d \equiv c$.

For more information on regular equivalence, the reader is referred to White and Reitz (1983) and Borgatti and Everett (1988).

We note that structurally equivalent vertices are automorphically equivalent, and automorphically equivalent vertices are regularly equivalent. The converses, however, are not true.

\section{Hierarchical approach to role analysis}

The approach we have in mind is illustrated in simplest form by the directed graph in Figure 1. REGE, the standard computer program designed to find the maximal regular equivalence, partitions the actors into three classes:

$\{a\}\{b, c, d\}\{e, f, g, h\}$.

For most analysts, this is the broadest and perhaps most intuitive partition. However, it is also clear that $b$ and $c$ are more similar to each other than they are to $d$, just as $e$ and $f$ are more similar to each other than to $g$ or $h$. This is certainly the case if you are interested in

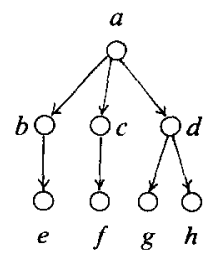

Figure 1. 
Table 1

\begin{tabular}{|c|c|c|c|c|c|c|c|c|}
\hline \multirow[t]{2}{*}{ Equivalence } & \multicolumn{8}{|c|}{ Vertex } \\
\hline & $a$ & $b$ & $c$ & $d$ & $e$ & $f$ & $g$ & $h$ \\
\hline Identity & & & . & . & . & . & . & \\
\hline Structural & . & & . & . & . & . & $x \times$ & $x \times$ \\
\hline Automorphic & . & & $x \times$ & . & & $x \times x$ & $x \times$ & $x \times$ \\
\hline REGE & . & & $\times \times$ & & & $x \times x$ & $x \times x$ & $x \times$ \\
\hline
\end{tabular}

Note: Singletons are indicated by full points.

perfect substitutability (Everett 1985), and are therefore concerned with degree. The appropriate measure of substitutability is automorphic equivalence, which partitions actors as follows:

$\{a\}\{b, c\}\{d\}\{e, f\}\{g, h\}$.

Of course, the strictest measure of role similarity is structural equivalence because it requires equivalent actors to be related to precisely the same people. Thus $\{g, h\}$ are more similar to each other than any other pair. The partition according to structural equivalence is as follows:

$\{a\}\{b\}\{c\}\{d\}\{e\}\{f\}\{g, h\}$.

Note that each of these models is hierarchically nested within the last. We can usefully display this information via the familiar dendrogram of hierarchical clustering, as shown in Table 1. For completeness, we have added the identity equivalence to the dendrogram, which is always regular.

We argue that by computing all of these models and placing them in their hierarchical context, a richer understanding of the structure of the graph is obtained. A side benefit of this approach is to emphasize that the differences between the three models discussed so far should be seen primarily in terms of the "coarseness" and "fineness" of the partitions they induce rather than in terms of different understandings of the role concept. All of the models belong to the family of regular equivalences and therefore embody the same general concept of role: in all models, two actors are said to play the same role if and only if they have the same relations with players of other roles. 


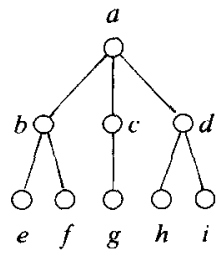

Figure 2.

Of course, there is no reason to be limited to the "standard" regular equivalences used above. A very useful equivalence is a "regularized" version of Winship and Mandel equivalence (for a discussion of regularization see Borgatti and Everett 1989). Whereas REGE equivalence always yields the trivial complete partition when applied to undirected graphs, Winship and Mandel equivalence (like automorphic equivalence) does not. And unlike automorphic equivalence, the Winship and Mandel model is not sensitive to degree. The regularized Winship and Mandel model is nested somewhere between automorphic equivalence and REGE equivalence.

More esoteric equivalences can also be used, such as the maximal regular equivalence preserving centrality. This and other equivalences generated by the REGE/A algorithm are described in Borgatti and Everett (1989).

For some graphs, however, none of the known equivalences will capture the intuitively best structure. Consider, for example, the undirected graph in Figurc 2.

Since it contains no sinks or sources, the maximal regular equivalence (which is the one computed by the standard program REGE) is the trivial complete partition. Obviously, in such a case we would like to use a less inclusive regular equivalence. The automorphic partition is as follows:

$\{a\}\{b, d\}\{c\}\{e, f, h, i\}\{g\}$.

The structural partition is:

$\{a\}\{b\}\{c\}\{d\}\{e, f\}\{g\}\{h, i\}$. 
None of these equivalences (including REGE) capture the intuitively best partitions, which are

No. $1\{a\}\{b, c, d\}\{e, f, g, h, i\}$

No. $2\{a, e, f, g, h, i\}\{b, c, d\}$.

An alternative approach is provided by the concept of iterated roles, which not only serves to generate new equivalences, but does so in such a way that the relationships between these equivalences is known.

\section{Iterated roles ${ }^{1}$}

Let $P$ be a partition of the vertex set $V$. The natural map $\tilde{n}: V \rightarrow P$ is defined for each $v \in V$ by letting $\tilde{n}(v)$ be the set in $P$ that contains vertex $v$. From $G$ construct a new graph $G^{\prime}$, called the image graph of $G$, with vertex set $P$ and edge set $R^{\prime}$ where $s R^{\prime} t$ iff there exists $a, b \in V$ with $a R b$ and $\tilde{n}(a)=s$ and $\tilde{n}(b)=t$. Hence $\tilde{n}$ can be thought of as a graph homomorphism from $G$ to $G^{\prime}$.

If we take the automorphic partition

$P_{1}=\{e, f, h, i\}$,

$P_{2}=\{g\}$,

$P_{3}=\{b, d\}$

$P_{4}=\{c\}$

$P_{5}=\{a\}$

of the graph in Figure 2, then the image graph $G^{\prime}$ is given by Figure 3 . If we now find the maximal automorphic equivalences for the graph in Figure 3, we obtain the partition

$\left\{P_{1}, P_{2}\right\}\left\{P_{3}, P_{4}\right\}\left\{P_{5}\right\}$,

1 The authors would like to note that Douglas $\mathrm{R}$. White has independently developed ideas similar in spirit to those contained here. We have benefited from his encouragement. 


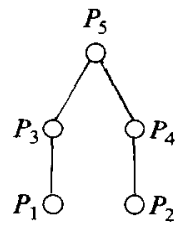

Figure 3.

which in terms of the original graph $G$ corresponds precisely to $\{a\}\{b, c, d\}\{e, f, g, h, i\}$.

Note that to obtain this partition we have employed only automorphic equivalence (albeit twice) and yet the result does not suffer from the limitation that members of a class have the same degree, as is suffered by single applications of automorphic equivalence. The first iteration gives substitutable individuals while the second gives substitutable classes. Iterated roles give a measure of degree of substitutability in the sense that vertices that are automorphically equivalent are primarilysubstitutable; but if only their automorphic equivalence classes are substitutable then the vertices are secondarily-substitutable, and so on.

Interestingly, taking automorphic equivalence one more time results in the partition

$$
\{b, c, d\}\{a, e, f, g, h, i\},
$$

which, it will be recalled, was previously described as one of the intuitively best partitions. To see why it is so desirable, redraw the graph in Figure 2 such that node $a$ is placed near $\{e, f, g, h, i\}$ : the result is the partition determined by the fact that the graph was bipartite.

We note that the partition of $V$ induced by secondary substitutability is itself a regular equivalence on $G$. In fact, any automorphic equivalence followed by another automorphic equivalence is always regular. Further, any regular equivalence of a regular equivalence is regular. We now prove this more general result.

Definition. Let $\tilde{n}: G \rightarrow G^{\prime}$ be defined as above. Then $\tilde{n}$ is a regular homomorphism (i.e. induces a regular partition) iff for all $a, b \in V$, 
$\tilde{n}(a) R^{\prime} \tilde{n}(b)$ implies there exists $c, d \in V$ such that $c R b, a R d$ and $\tilde{n}(c)=\tilde{n}(a)$ and $\tilde{n}(d)=\tilde{n}(b)$. A proof of this result is found in White and Reitz (1983).

Theorem 1. Let $\tilde{n}: G \rightarrow G^{\prime}$ be regular and $\tau: G^{\prime} \rightarrow G^{\prime \prime}$ be regular, then $\tau \cdot \tilde{n}$ is regular.

Proof. Suppose $\tau \cdot \tilde{n}(a) R^{\prime \prime} \tau \cdot \tilde{n}(b)$. Since $\tau$ is regular then there exists $\tilde{n}(c)$ and $\tilde{n}(d)$ such that $\tilde{n}(c) R^{\prime} \tilde{n}(b), \tilde{n}(a) R^{\prime} \tilde{n}(d), \tau \cdot \tilde{n}(c)=\tau \cdot \tilde{n}(a)$, and $\tau \cdot \tilde{n}(d)=\tau \cdot \tilde{n}(b)$. Since $\tilde{n}$ is regular and $\tilde{n}(c) R^{\prime} \tilde{n}(b)$ then there exists $e, f$ such that $e R b, c R f, \tilde{n}(e)=\tilde{n}(c)$ and $\tilde{n}(f)=\tilde{n}(b)$. Since we also have $\tilde{n}(a) R^{\prime} \tilde{n}(d)$ then there also exists $g$ and $h$ such that $g R d$, $a R h, \tilde{n}(g)=\tilde{n}(a)$ and $\tilde{n}(h)=\tilde{n}(d)$.

Hence we have that $e R b$ and $a R b$ with $\tau \cdot \tilde{n}(a)=\tau \cdot \tilde{n}(c)$, but since $\tilde{n}(c)=\tilde{n}(e)$ it follows that $\tau \cdot \tilde{n}(a)=\tau \cdot \tilde{n}(e)$. Also $\tau \cdot \tilde{n}(b)=\tau \cdot \tilde{n}(d)$ but $\tilde{n}(d)=\tilde{n}(h)$ so that $\tau \cdot \tilde{n}(b)=\tau \cdot \tilde{n}(h)$. Therefore $\tau \cdot \tilde{n}$ is regular.

Corollary. Iterated automorphic equivalence is regular.

Taking a regular equivalence of another regular equivalence provides a means of simplifying the initial model, generating a new model that is coarser than the first without contradicting it. The graph in Figure 3 is a simplification of the original graph in Figure 2. In Figure 4, we take the simplification process to its logical extreme, ending with the maximal regular equivalence where all vertices are equivalent. For the series in Figure 4 (summarized in the dendrogram of Table 2), we chose automorphic equivalence as the simplifying operation at each step. Clearly, the degree of simplification obtained will vary depending on which regular equivalences are chosen. A maximal regular equivalence followed by maximal regular equivalence will by definition provide no simplification. Thus, running REGE on the results of REGE will accomplish nothing. In fact, any regular equivalence followed by REGE will be the same as applying REGE directly, and any regular equivalence applied after REGE has no effect.

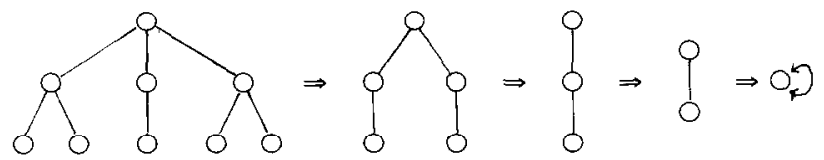

Figure 4. 
Table 2

Automorphic reduction for graph in Figure 2.

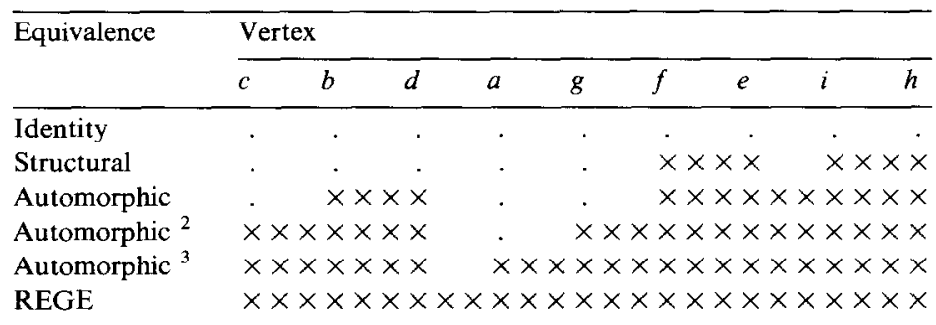

Note: Full points indicate singletons.

Obviously, taking structural equivalence of a structural equivalence gives a structural equivalence. However, this will not produce any equivalences which were not deducible directly from the original graph. Once all pairs of structurally equivalent vertices are known then the complete lattice of structurally equivalent vertices can be deduced. In addition, iteration of maximal structurally equivalent vertices is not possible as we can see from the following theorem.

Theorem 2. Let $\tilde{n}: G \rightarrow G^{\prime}$ be a maximal structural equivalence. Then $G^{\prime}$ contains no structurally equivalent vertices.

Proof. Let $P_{i}$ and $P_{j}$ be two structurally equivalent vertices in $G^{\prime}$. We shall prove that the elements of $P_{i}$ are structurally equivalent with the elements of $P_{j}$, contradicting the maximality of $\tilde{n}$. Let $a \in P_{i}$ and $b \in P_{j}$, and suppose $a R c$ where $c \in P_{k}$. Then $P_{i} R^{\prime} P_{k}$. Since $P_{j}$ is structurally equivalent to $P_{i}$, we have that $P_{j} R^{\prime} P_{k}$. Hence there exists $d \in P_{j}$ and $e \in P_{k}$ with $d R e$. Since the elements of $P_{j}$ are structurally equivalent we must have that $b R e$. Also, since the elements of $P_{k}$ are structurally equivalent then $b R c$. Hence, whenever $a R c$ then $b R c$. A similar argument shows that whenever $d R a$, then $d R b$ so that $a$ and $b$ are structurally equivalent and the result follows.

Hence the only combinations of the three well-known regular equivalences to yield new equivalences are: automorphic with automorphic and automorphic with structural. There are, however, a variety of other regular equivalences that could be used to find iterated regular equivalences. Any equivalence generated via REGE/A, for example, may be used either as a starting point or as a simplifying operation. 


\section{Iterated automorphic equivalence}

In this section we consider paths through the lattice generated by repeated applications of automorphic equivalence. Consider again the reduction series shown in Figure 4 and Table 2. In this case, the iterative process yields quite sensible results at every step. Some graphs, however, pose a problem. Consider, for example, the series in Figure 5. Here, one component has one more branch than the other, and so the procedure fails to notice the significant similarities between the components.

The situation is remedied by a simple intervention on the part of the analyst: iterating the larger component separately. A single iteration of the left component yields a new structure identical to the right component, and now iterating the entire graph yields the more intuitive results shown in Figure 6.

This technique works in general and any component or group of components can be iterated separately. When iterating components separately the maximum cross-component groupings will occur if the largest components are iterated first, followed by an iteration of the entire graph.

The ability to iterate components separately is an example of a more general principle that allows subregions of a graph to be iterated separately. In Figure 6, the reason that we could collapse the leftmost branches of the graph separately was that a regular equivalence (indeed, an automorphism) existed that sent the vertices of one branch onto the vertices of the other, while fixing all other points of the graph. We can, of course, use any regular equivalence which groups together desired vertices while leaving others fixed. The difficulty is in finding such an equivalence. A method based upon automorphic equivalence is described next.

Consider the orbits of any subgroup of the automorphism group of a
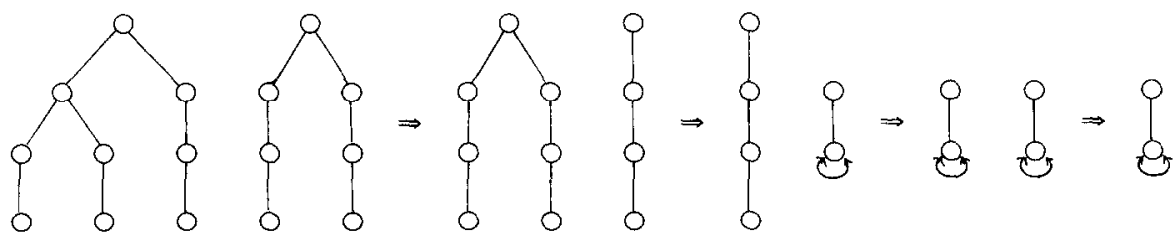

Figure 5. 


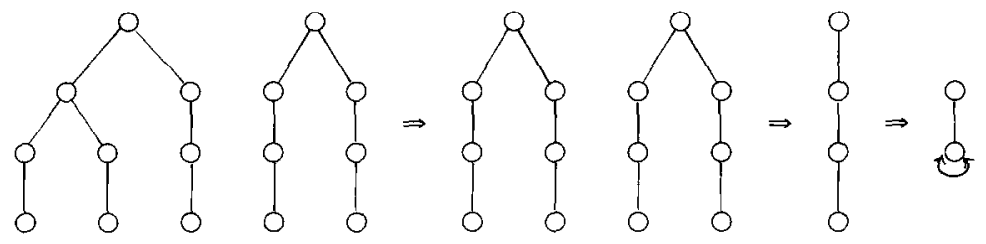

Figure 6.

graph. These form a regular equivalence. Obviously the orbits of any connected component of $G$ will be the orbits of a subgroup of $\operatorname{Aut}(G)$ (it is the one fixing those vertices of $G$ not in the component) and it is for this reason that we can iterate components separatcly. (However it is not the case that all orbits can be iterated separately.)

An approach to finding useful subgroups of $\operatorname{Aut}(G)$ is to use one of the permutations as a generator. In this case, the elements of the cycle will be precisely the orbits of the subgroup generated by the permutation. For example, suppose we wish to collapse vertices 1 and 5 of the graph in Figure 7 prior to iterating the rest of the graph. We need to find an automorphism which maps 1 to 5 but fixes as many other vertices as possible. Obviously, (1 5)(26) is such an automorphism, and no "smaller" automorphism (i.e. one fixing more points) exists. Thus to collapse 1 and 5 we must also collapse 2 and 6 in order to ensure that the resulting partition will be regular.

We note that since two vertices $a, b \in V$ are structurally equivalent if and only if $\pi=(a b)$ is an automorphism, we can always group together non-maximal sets of structurally equivalent vertices to create new regular equivalences; we need not collapse all of them. Thus in Figure 8 , we are free to collapse only $\{a, b\}$ and $\{c, d\}$, thus allowing the next iteration to "see" the correspondence between points across components.

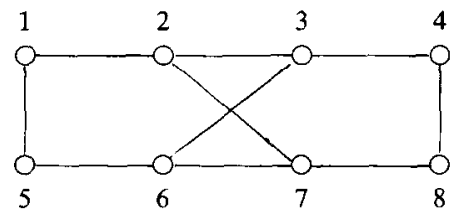

Figure 7.
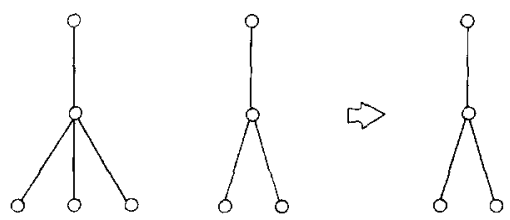

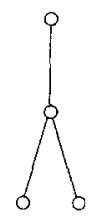

Figure 8. 
It should be clear that most graphs will have a number of subregions that may be iterated separately, particularly if we consider not just automorphism orbits but all regular partitions. Each decision to iterate or not to iterate a region independently gives rise to a new path through the lattice. Each element of such a path is itself a graph with subregions, each of which may be iterated separately or in combination. The lattice itself may be viewed simply as the set of all combinations of these independent decisions.

Another problem that can occur in the analysis of real data is that there are not automorphically equivalent vertices and so the process fails to get started. In this case, we can start the process with a more liberal, more abstract regular equivalence. If even the maximal regular equivalence fails to give any simplification, a non-regular equivalence such as local regular equivalence (Everett et al. 1988) may be attempted. The iterative procedure is then applied to that result, keeping in mind that the resulting sequence of models will not be regular equivalences. Alternatively, ad hoc procedures may be employed, such as collapsing the two most similar points and restarting the iterations.

\section{Discussion}

From a theoretical point of view, the iterated roles procedure provides a tool for exploring the lattice of regular equivalences. From a methodological point of view, iterated roles provide a way of viewing increasingly broad simplifications of the role structure of a graph. In this sense, it is very much like performing a hierarchical clustering such as advocated by Burt (1976). Burt computes a distance matrix consistent with a particular model of role (in his case, structural equivalence), and then submits it to hierarchical clustering, which generates a series of nested partitions of the vertices. The principal difference between this and iterated roles is that in Burt's approach the partitions are generated by an atheoretical procedure (clustering), so none of the resultant partitions need conform to any formal model of role. In contrast, every partition in an iterated role analysis is a regular equivalence, and hence a model of role. A related distinction is that in Burt's method, the hierarchical nesting is found in the pattern of deviations of data from an extremely strict theoretical model; in iterated roles, the nesting 
results not from the data but from the theoretical models, which are nested.

Computer programs for performing itcrated role analyses are available as part of the NETPAC suite of network programs (Borgatti 1988).

\section{References}

Arabic, Phipps and Scott A. Boorman 1982 "Blockmodels: Developments and Prospects". In Herschel Hudson (ed.) Classifying Social Data. San Francisco: Jossy-Bass.

Arabie, Phipps, Scott Boorman, and P.R. Levitt

1978 "Constructing Blockmodels: How and Why". Journal of Mathematical Psychology 17: $21-63$.

Boorman, S.A. and White H.C.

1976 "Social Structures from Multiple Networks: II. Role Structures". American Journal of Sociology 81 (May): 1384-1446.

Borgatti, Stephen P.

1988 User's Manual for NETPAC. Irvine: University of California.

Borgatti, S.P. and M. Everett

1989 "The Class of all Regular Equivalences: Algebraic Structure and Computation". Social Networks (in press).

Boyd, John P. and M.G. Everett

1988 "Block Structures of Automorphism Groups of Social Relations". Social Networks 10: 137-155.

Breiger, R.L., S. Boorman, and P. Arabie

1975 "An Algorithm for Clustering Relational Data with Applications to Social Network Analysis". Journal of Mathematical Psychology 12: 329-383.

Breiger, R.L., and P.E. Pattison

1986 "Cumulated Social Roles: The Duality of Persons and their Algebras". Social Networks 8: 215-256.

Burt, R.S.

1976 "Positions in Networks". Social Forces 55: 93-122.

1982 Toward a Structural Theory of Action: Network Models of Social Structure, Perception, and Action. New York: Academic Press.

Everett, M.G.

1985 "Role similarity and complexity in social networks". Social Networks 7: 353-359.

Everett, M.G. and S.P. Borgatti

1988 "Calculating Role Similarities: An Algorithm Which Helps Determine the Orbits of A Graph". Sacial Networks 10: 77-91.

Everett, M.G., S.P. Borgatti and J.P. Boyd

1988 "Ego-Centered and Local Roles: A Graph Theoretic Approach". Journal of Mathematical Sociology (in press).

Lorrain, F.P. and H.C. White

1971 "Structural Equivalence of Individuals in Networks". Journal of Mathematical Sociology I (January): 49-80.

Mandel, Michael J.

1978 "Roles and Networks: A Local Approach". Senior honors thesis, Harvard University.

1983 “Local Roles and Social Networks". American Sociological Review 48: 376-386. 
Mandel, Michael J. and Christopher Winship

1979 "Roles, Positions and Networks". Presented American Sociological Association meetings, Boston.

Sailer, Lee D.

1978 "Structural Equivalence: Meaning and Definition, Computation and Application". Social Networks 1: 73-90.

White, D.R. and K.P. Reitz

1983 "Graph and Semigroup Homomorphism on Networks of Relations". Social Networks 5: 193-235.

1984 "REGGE: A Regular Graph Equivalence Algorithm For Computing Role Distances Prior to Blockmodeling". Unpublished manuscript. University of California, Irvine.

White, H.C., S.A. Boorman and R.L. Breiger

1976 "Social Structure from Multiple Networks: I. Blockmodels of Roles and Positions". American Journal of Soriolngy 81 (January): 730-80.

Winship, C.

1974 "Thoughts about Roles and Relations". Unpublished paper, Department of Sociology, Harvard University.

Winship, C. and M.J. Mandel

1983 "Roles and Positions: A Critique and Extension of the Blockmodeling Approach". In S. Leinhardt (ed.) Sociological Methodology 1983-84. San Francisco: Jossey-Bass. 